\title{
Deleuze: una ontología, una ciencia
}

Gonzalo Ramírez Herrera

Pontificia Universidad Católica del Perú

Resumen: La presente contribución tiene dos objetivos. En primer lugar, rebatir tres visiones equivocadas sobre la filosofía de Gilles Deleuze. Estas visiones giran en torno a su posición en ontología, en estética y en ciencia. En segundo lugar, profundizar en dos de estos malentendidos con el fin de descubrir sus alcances actuales. Estos dos malentendidos son sobre su visión ontológica y su visión sobre la ciencia. Acá veremos cómo se plantea una ontología de la diferencia y una ciencia intensiva. Cerraremos nuestra contribución con algunos comentarios sobre la actualidad de este pensamiento.

Palabras clave: ontología, estética, ciencia, devenir, différence.

Abstract: This paper has two objectives: first, to rebut three misconceptions about the philosophy of Gilles Deleuze that deal with his position in ontology, aesthetics and science; and second, to probe deeper into two of these misunderstandings in order to discover their current scope. Both of these misunderstandings regard his views on ontology and on science. Here we will see how an ontology of difference and an intensive science arise. We will close our paper with some comments on the actuality of this thought.

Keywords: ontology, aesthetics, science, becoming, différence. 


\section{§ 1. Introducción}

Gilles Deleuze es caracterizado por Alan Badiou en la obra titulada Deleuze, clamor del ser como "el inventor de un Barroco contemporáneo, donde nuestro deseo de multiplicidad, de mestizaje, de coexistencia de universos sin regla común, en suma, (donde) nuestro democratismo planetario, encuentran un lugar donde reflejarse y desplegarse"'. Aquí se retrata a "Deleuze como pensador alegre de la confusión del mundo" y donde "su doctrina alienta la multiplicidad heterogénea de los deseos e incita su realización sin trabas; que se preocupa por el respeto y la afirmación de las diferencias; que constituye por esta razón una crítica conceptual de los totalitarismos (...)"2. El propio Badiou reniega de esta caracterización, y nosotros también. Si bien esta es una visión clásica, por no decir cliché, de este autor, no agota la importante propuesta deleuzeana o en todo caso la equivoca $y$, además, alienta tres malentendidos que revisaremos en esta intervención. En estos se le tilda de posmoderno, de esteticista y de anticientífico. Revisemos ${ }^{3}$ :

a) Deleuze, indica el prejuicio, al ser un pensador postmoderno, un postmetafísico, se desentiende de cualquier cuestión ontológica. Esto, a su vez, implica que se asuma a su obra como "asistemática, inmediata, espontánea y que su pensamiento encarna la reactualización al peor culto a la indeterminación romántica, a la vaguedad y a lo indiferenciado"4.

b) Asimismo, se asume que en cuestiones prácticas, Deleuze es un "esteticista”, es decir, un nuevo gurú del buen vivir, un relativista en ética, y en el ámbito de la clínica, un mero contradictor del psicoanálisis.

c) En cuestiones epistemológicas, se indica, su pensamiento comporta una profunda desvalorización de lo científico o que se halla por completo alejado de las problemáticas propias de la ciencia. Para algunos, es un profundo "antipositivista".

Sobre el punto a), el que lo tilda de posmoderno podemos decir lo siguiente. La filosofía de Gilles Deleuze es todo menos asistemática: es un sistema, sin

I Badiou, Alain, Deleuze, el clamor del ser, Manantial: Buenos Aires, 1997, p. 21.

2 Ibid.

3 Cf. Hardt, Micheal, Gilles Deleuze. Un aprendizaje filosófico, traducción de Alcira Bixio, Paidós: Buenos Aires, 2004, pp. 19-20.

I44 4 lbid. 
duda, pero es un sistema en tanto heterogéneo, y ante todo, despliega una lógica de lo múltiple. La crítica que practica Deleuze de los sistemas cerrados, no criticaba la sistematicidad que dibujaban sino su carácter totalizante, cerrado y no productivo ${ }^{5}$. Es decir, podemos (es más, debemos) buscar otra idea de sistema en tanto principio de conexión. Así ya no tenemos un sistema de lo cerrado, sino de lo abierto, de la conectividad. En este sentido, no se abandona el ideal, sino el antiguo emplazamiento hegeliano. Ahora bien, lo abierto deleuzeano implica lo indeterminado. Lo indeterminado es el caos. Pero el caos, y aquí el detalle, no es lo opuesto a cualquier determinación sino que es leída por Deleuze como aquella instancia que, en tanto encarna una potencia absoluta de diferenciación, tiende a hacer posible la existencia de algo tal como un determinado. Es lo que se veía en los libros sobre cine ${ }^{6}$ como la imagen-potencia. Lo indeterminado no es así lo contrario de lo determinado sino su condición, aquel ámbito ontológico que hace posible cualquier determinación y que permite a los determinados existir unos junto a otros aun cuando resulten incapaces de componerse. Deleuze ha sido uno de los pensadores del siglo $X X$ que más se ha comprometido con la tarea de seguir pensando en términos ontológicos. Sin embargo, su interés está en elaborar una ontología del devenir', es decir, subsumir al ser en el devenir y pensar a la diferencia (différance) como la única realidad del ser. En Deleuze, nos encontramos ante una reformulación, entonces, de las condiciones bajo las cuales lo indeterminado se relaciona con la determinación y lo determinado.

Con respecto al punto b), debemos indicar que Deleuze no es un esteticista: no desvaloriza el dolor, no invita a distraerse, tampoco a participar de un culto de la comodidad y de lo agradable. Más bien, la estética deleuzeana es una preocupación por las condiciones de sensibilidad; una atención que sin duda se preocupa por el arte, pero solo en tanto este último se constituye como caso privilegiado para el análisis de aquellas situaciones en que la receptividad y la sensibilidad se presentan como el grado cero de una cierta creación singular. La cuestión no es cómo se accede o contempla la belleza, sino generar diversas formas de sentir a través del análisis de estas modalidades. Entendida de

5 Cf. Deleuze, Gilles, El bersognismo, Cátedra: Madrid, 1987, pp. 5I-52.

6 Cf. Deleuze, Gilles, Estudios sobre cine. La imagen-movimiento, Paidós: Barcelona, 1984, p. 151.

7 Cf. Deleuze, Gilles, Lógica del sentido, Paidós: Barcelona, 2005, p. 98. 
esta manera, la estética no se opone a la acción: la acción es un derivado de aquella sensibilidad que necesariamente se constituye en torno de cualquier región donde el ser del devenir se afecta a sí mismo. Esta revisada visión de estética conduce directamente a la noción de jovialidad (joie). La jovialidad no es estar a gusto, no es sentirse satisfecho y logrado, no es la negación de la muerte, sino la capacidad del deseo y de la vida para sobreponerse a los efectos destructivos de la misma. Es una condición para disponer de la mayor capacidad de acción y de respuesta ante la vida.

Con respeto a c), al prejuicio anti-cientificista que se cierne sobre Deleuze, debemos indicar que nuestro autor busca volver a pensar la ciencia y dar a la verdad el lugar que merece. Deleuze nunca se ha mostrado como negador de la verdad. Se suele indicar que para Deleuze hay cuestiones más importantes que la verdad o en todo caso que él busca promover otra concepción de verdad, una en la cual esta sea derivada no de lo verdadero en el mundo, sino de la creación como potencia ontológica -y lo creado no es lo que se manifiesta a nivel proposicional o un juicio determinado, sino lo que permite a esta verdad aparecer como verdad entre otras. Lo que busca acá Deleuze es tratar de pensar de modo distinto estas condiciones para hacer surgir la verdad en tanto campo de posibilidades. Quizá el concepto que mejor permita hablar de estas condiciones sea el de "plano de inmanencia": un plano presupuesto donde las ideas del pensador pueden construirse y tomar conciencia. Es sobre este todo no totalizante, siempre productivo, que vienen a repartirse los conceptos y se produce la creación filosófica y científica. Este uno-todo es como un desierto móvil que los conceptos vienen a poblar sin dividirlo: es un campo donde la materia es la intuición.

Estas deben ser las tres líneas de defensa de este autor. Más que de defensa, de redescubrimiento, ya que este filósofo es uno de los principales renovadores del pensamiento actual. Deleuze es una vuelta a la novedad del pensamiento en sí mismo. Como no va a serlo, si la filosofía es, como él la concibe, el arte más puro: el arte de crear, crear conceptos.

Nuestro trabajo consistirá en abordar, panorámicamente, tanto a) como c). En a) caracterizando sus fundamentos ontológicos y en c) presentando posible vías para una ciencia desde el punto de vista deleuzeano. Sobre este último punto nos detendremos en ejemplos de ciencias que integren características 
del pensamiento deleuzeano en su despliegue. No olvidemos que esto es ante todo una presentación de nuevos caminos a pensar y sobre todo a discutir.

\section{§ 2. Ontología}

Los trabajos de Deleuze son un desarrolloy aprendizaje filosófico: un recorrido donde la lectura de diversos autores consolida, por decirlo de alguna manera, una vía alternativa a los problemas clásicos del pensamiento filosófico. Pero este camino no es de saltos bruscos ni cortes o hiatos, es más bien de vueltas y revisiones donde la atención y el momento del autor guían la lectura. El primer eje de este camino es, sin duda, Henri Bergson ${ }^{8}$. Deleuze encuentra en este autor una lógica absolutamente positiva del ser arraigada en el tiempo: el terreno que más adelante servirá para inscribir el binomio actual-virtual. Desde el punto de vista de Deleuze, Bergson concentra su crítica en el movimiento ontológico que describe Hegel, quien puede ser considerado el principal antagonista a combatir por ser el terreno clásico donde la filosofía de fines del siglo XIX transitaba. Parece suponerse que al criticar esta noción, la de movimiento ontológico, se renovarían, casi como en un efecto dominó, las nociones clásicas de la ontología como perfección, unidad, multiplicidad, etc. Es ahí donde Deleuze comienza su trayecto.

Desde la lectura de Deleuze, la "différence"” es el concepto central en la crítica al movimiento ontológico. Esta noción no se refiere a una distinción sutil: la diferencia marca la dinámica real del ser, es el movimiento que sustenta al ser. Deleuze indica: "La diferencia no es lo diverso. Lo diverso está dado. Pero la diferencia es aquello mediante lo cual lo dado es dado. Es aquello mediante lo cual lo dado es dado como diverso. La diferencia no es el fenómeno, sino que está más cercana del nóumeno que del fenómeno. Es, pues, bien cierto que Dios hace al mundo mediante cálculo, pero sus cálculos no dan nunca en lo justo, y es esa injusticia en los resultados, esa irreductible desigualdad la que conforma la condición del mundo. El mundo «se hace» mientras Dios calcula; no habría mundo si el cálculo fuera justo. El mundo es siempre asimilable a un «resto», y lo real en el mundo sólo puede ser pensado en términos de

8 Cf. Bergson, Henri, Materia y memoria, Editorial Cactus: Buenos Aires, 2006, p. 88.

9 Cf. ibid. 
números fraccionarios o incluso inconmensurables. Todo fenómeno remite a una desigualdad que lo condiciona. Toda diversidad, todo cambio, remiten a una diferencia que es su razón suficiente"10.

En este sentido, la diferencia transciende a la comparación empírica entre dos objetos particulares. Estamos tratando de describir a la diferencia misma. Sobre esta misma idea, se debe seguir que la différence a la que aquí nos remitimos es una potencia interior al ser, autónoma, independiente que contiene su propio germen de desarrollo.

Son dos tareas las que Deleuze le asigna a este concepto: I) poner de relieve la debilidad de la dialéctica hegeliana y su lógica negativa como una falsa concepción del ser. La segunda tarea es 2) elaborar el movimiento positivo del ser: mostrar una alternativa viable a la ontología y preparar el terreno para el momento productivo.

La primera tarea consiste en criticar a dialéctica negativa. Para entender la crítica hacia Hegel primero, como es lógico, hay que acercarnos a Hegel. Este recorrido será sobre el primer paso dentro del despliegue hegeliano: la certeza sensible. Esto por ser, consideramos, un mismo punto de encuentro y de despegue entre Hegel y Bergson. Para Hegel la existencia de algo es la negación activa de alguna otra cosa. La determinación es así el proceso y el estado del ser determinado. La lógica comienza con el ser puro en su simple inmediatez; pero este ser es simple, no tiene ninguna cualidad, ninguna diferencia: es vacío y es todo a la vez, por ello, es equivalente a su opuesto, la nada. Es necesario que el ser niegue activamente la nada para marcar diferencia con ella. El ser determinado en esta negación subsume esta oposición entre el ser y la nada y define el fundamento de las diferencias y cualidades que constituyen la realidad del ser. Se indica que la negación define este estado del ser en dos sentidos: es un contraste estático basado en la finitud de las cualidades y un conflicto dinámico basado en el antagonismo de las diferencias.

Por consiguiente hasta el estado de algo es un movimiento negativo. En términos del propio Hegel: "El contenido concreto de la certeza sensible hace que esta 
se manifieste de un modo inmediato como el conocimiento de riqueza infinita a la que no es posible encontrar límite si vamos más allá en el espacio y en el tiempo en el que se despliega (...). Este conocimiento se manifiesta, además como el más verdadero, pues aún no ha dejado a un lado nada del objeto si no que lo tiene ante sí en toda su plenitud. Pero de hecho, esta certeza se muestra ante sí misma como la verdad más abstracta y más pobre""l.

Hasta este punto, salvo la última idea donde el ser en sí mismo es tan abstracto que equivale a la nada, estaría de acuerdo con lo que sostiene Bergson, pero Hegel prosigue: "En ella lo uno está puesto como lo que es de un modo simple e inmediato o como la esencia, es el objeto; lo otro lo está como lo no esencial y mediado, que es allí no en sí, sino por medio de otro, el yo, un saber que solo sabe el objeto porque él es y puede ser o no ser"'2.

Acá diera la impresión que se necesita de un agente externo para dar cuenta del denominado movimiento ontológico. En todo caso, esa es la lectura canónica a la que Bergson ataca y, creemos, retrata bien la situación: el despliegue dialéctico clásico implica la presencia de más elementos que solo el Ser en su estado de pureza.

Es lógico pensar que para Hegel cualquier intento de ontología positiva, donde el Ser no niega activamente nada y no procede mediante un movimiento negativo, será una ontología que se diluirá sola en su propio campo abstracto e indiferenciado. El Ser no diferenciado se disipara en la nada, como le critica a Spinoza' ${ }^{13}$.

Deleuze arremete contra el proceso de determinación ontológico mismo y sostiene que este socava el fundamento real del ser $y$ afirma que la diferencia constituida por el movimiento negativo de determinación es una falsa noción de diferencia. En este mismo sentido, afirma, que el proceso de determinación no solo destruye la naturaleza sustancial del Ser, además no comprende el carácter concreto ni la especificidad del Ser real.

II Helgel, G.W.F., Fenomenología del espíritu, traducción de Wenceslao Roces y Ricardo Guerra, México D.F.: Fondo de Cultura Económica, 2008, p. 63.

12 Ibid., p. 74.

I3 Cf. Artola, José María, “La crítica hegeliana a la filosofía de Spinoza”, en: Logos: Anales del Seminario de Metafísica, No. I (1992), pp. 635-656. 
¿Cuál es el error principal de esta posición, según Deleuze? Deleuze indica que para que el Ser sea necesario, debe ser indeterminado. Una determinación mecanicista del Ser, la cual permanece externa, no puede proporcionarle un fundamento esencial necesario. Acáindeterminado antes que abstracto ogaseoso debe entenderse como independiente de cualquier tipo de determinación impuesta desde la exterioridad. La diferencia externa de determinación siempre depende de otro, como causa o como fin, y por lo tanto, introduce una cualidad accidental en el ser: implica así una exterioridad subsistente, no una interioridad sustancial.

Deleuze atribuye a la diferencia un rol radicalmente nuevo. La diferencia otorga fundamento al ser, le suministra su sustancialidad y su necesidad. Es decir, no perseguimos causas per accidens, sino causas per se. Lo que debe quedar en claro es que Deleuze no establece una crítica contra todo tipo de causalidad: solo contra las que implican exterioridad, quedándose así solo con las causas internas ${ }^{14}$.

En resumen, esta primera cara de la ontología deleuzeana se sustenta en Bergson y en su noción de diferencia donde se define como el principio del movimiento positivo del ser en un plano temporal. Esta diferencia al ser interna se distingue de la diferencia mecanicista donde la "causa" es material y accidental, es decir, externa.

La diferencia bergsoniana debe distinguirse además de la diferencia platónica que se funda, no en una causa material, sino en una causa final. Este movimiento es igualmente externo porque está fundado por una causa exterior a la naturaleza interna del ser. En este sentido, la diferencia hegeliana, que es la diferencia mecanicista y platónica llevada a un nivel más radical, ya que es una causa abstracta en el sentido de que el movimiento negativo que propone es una causa absolutamente externa a su efecto, es la que Deleuze, de la mano de Bergson, más rechazará. La crítica es más fuerte aún, como hemos visto: esta oposición radical, la hegeliana, es una noción demasiado simplista para captar los matices que marcan las diferencias reales. 
La diferencia de Bergson se debe definir mediante una noción de causalidad interna. El movimiento del ser es una progresión de diferencias internas por cuanto la causa es siempre inherente a su efecto. Es así que el ser se libera del juego de las negaciones y se presenta positivo.

La segunda tarea consiste en elaborar el movimiento positivo del ser. La positividad del ser y aceptar que el mismo es indeterminación es de por sí una refutación del movimiento negativo de la dialéctica. En 1960, Deleuze publica El bersognismo ${ }^{15}$ y si bien ese texto mantiene el ataque contra este tipo de dialéctica, el eje de discusión es ahora el problema de lo uno y lo múltiple. Veamos. En este texto se indica que el movimiento negativo de la dialéctica viola las relaciones reales del ser, ya que es un movimiento falso, un movimiento del concepto abstracto que va de un opuesto a otro solo mediante la imprecisión. Sin embargo, aquí Deleuze encuentra más que un problema ontológico, un problema político: traslada la ontología a la esfera política. Para Hegel es evidente que la relación de lo uno y lo múltiple es un fundamento para una teoría de la organización social, una base ontológica para la política' ${ }^{16}$. Esto es como decir que la doctrina del arché es una ontología base para una tiranía: el gobierno de uno sobre distintas multiplicidades. Atacar la unidad dialéctica de lo uno y lo múltiple es pues atacar la primacía del Estado en la formación de la sociedad. Es negar la pluralidad real de la sociedad. Opta, entonces, por hablar de multiplicidad en vez de lo uno y lo múltiple. Así, lo múltiple externo es una multiplicidad de orden, la multiplicidad interna es una multiplicidad de organización. Deleuze defiende esta última, un pluralismo de la organización contra un pluralismo del orden. Como indica Alain Badiou, lo uno ni lo múltiple no existe para Deleuze, pues ambos conceptos no pueden explicar la confusión del mundo. El mundo no se encuentra en movimiento ni en el régimen de lo estable, por lo que se debe de renunciar a la concepción del ser según lo uno y lo múltiple. El uno más bien se debe de entender con el nombre de "Uno-todo". El ser, afirma Deleuze, es el único acontecimiento en el que todos los acontecimientos se comunican, por lo tanto se puede decir que el ser es el sentido. El ser es el uno en cuanto un acontecimiento para todos, esto es, un solo ser para lo imposible, lo posible y lo real, llegar a este

I5 Para comprender la visión que Deleuze adopta de Bergson también puede verse: Bergson, Henri, Memoria y vida: textos escogidos por Gilles Deleuze, Madrid: Alianza Editorial, 2004.

16 Cf. Badiou, Alain, Condiciones, México D.F.: Siglo XXI, 2002, p. 206. 
"un solo" es el trasfondo real de lo que se puede denominar como deseo, pues "todo viene siempre de más lejos, y es más: todo está ahí, en los recursos infinitos e inhumanos del Uno" ${ }^{17}$.

Los términos de la alternativa ya quedaron establecidos por la crítica: a través de un movimiento positivo, interno, el ser debe llegar a ser (devenir) calificado y concreto en su singularidad y su especificidad. Aquí es donde surge la noción de virtual como la simplicidad del ser, puro, que obtiene cualidades a través del proceso interno de diferenciación. Aquí, entonces, la tarea principal es elaborar el movimiento positivo del ser entre lo virtual y lo actual. Esto sustenta la necesidad del ser y le proporciona identidad y al mismo tiempo diferencia; unidad y al mismo tiempo multiplicidad. La duración (durée) ${ }^{18}$ es el ámbito en el cual inscribimos el movimiento ontológico primario: la duración, esteámbito del tiempo cualitativo que describe Bergson, puede diferir cualitativamente de sí misma. El espacio, el movimiento espacial no puede diferir de sí mismo, antes bien se repite.

Toda esta descripción lo que busca es inscribir al ser dentro de un terreno donde este pueda diferir propiamente de sí: este es un espacio temporal, es la duración. Ahora bien, el movimiento ontológico de lo virtual a lo actual es coherente con el hecho de que un efecto no puede tener más realidad que su causa, ya que lo virtual es tan real como lo actual. En este mismo sentido, observamos que las reglas que gobiernan el proceso de actualización son la diferencia y la creación, y no la semejanza y la limitación como en el caso del paso de lo posible a lo real, elementos de distinto plano y de distinta realidad.

Acá añadiremos unos comentarios más sobre lo que toma Deleuze de Spinoza. No serán muy extensos por lo complejo de este autor, pero intentaremos dar algunos alcances para terminar de caracterizar al ser que Deleuze intenta mostrar. Deleuze considera el sistema de Spinoza como dos momentos distintos: uno especulativo y otro práctico, que es el mismo paso de la afirmación a la alegría ${ }^{19}$. Sobre el primer punto, hay que partir por el hecho de que las propuesta de Bergson y Spinoza suelen vincularse con facilidad por implicar

17 Badiou, Alain, Deleuze, el clamor del ser, p. 24.

I8 Cf. Bergson, Henri, op. cit., p. 15.

15219 Cf. Deleuze, Gilles, Spinoza. Filosofía práctica, Buenos Aires: Tusquets, 2004, p. 135. 
la descripción de un ser positivo y de la causalidad interna al mismo. Para Spinoza, por ejemplo no es posible equiparar el ser con el número porque este último implica una limitación y, por lo tanto, una causa externa. Esto a su vez nos presenta un ser inmerso en el absoluto. Causa sui es entonces, en Spinoza, que el ser es infinito e indivisible, al tiempo que es distinto y está determinado. Asimismo, la positividad del ser se caracteriza por su singularidad y su expresión unívoca. El ser spinoziano es notable, como indica Hardt, ya que es diferente sin ninguna referencia externa. Así como el ser es causa de sí mismo y, por lo tanto, está sustentado por una estructura causal interna también es diferente en sí mismo: se sostiene a través de una noción de diferencia interna. La expresión de esta diferencia interna es precisamente el movimiento positivo del ser.

Cuando Deleuze se refiere a la univocidad del ser, no se refiere a que el ser sea numéricamente uno, ni al ser en relación a lo uno como identidad. Más bien se afirma que la potencia del uno significa que los entes son múltiples y diferentes, disyuntivos y divergentes, pues se da la existencia de múltiples formas del ser. Es en la potencia donde se encuentran las múltiples formas del uno, pues "el Ser se dice en un solo y mismo sentido de todas las formas (...) los atributos inmanentes del Ser, que expresan su infinita potencia en tanto Uno, son formalmente distintos, pero todos iguales y ontológicamente uno"20. Las múltiples acepciones del ser son distinciones formales. En cada forma del ser se pueden dar diferencias individuantes, que se han de entender como entes. Para Deleuze, los entes son grados locales de intensidad o reflexiones de la potencia que se encuentran constantemente móviles y son enteramente singulares, " $y$ como la potencia es sólo un nombre del Ser, los entes no son sino modalidades expresivas del Uno. De ahí que la distinción numérica entre entes resulte una distinción modal, y nunca real”2!. Está claro que el objetivo de fondo de esta crítica apunta a la determinación del ser y a la dialéctica de lo uno y lo múltiple.

20 Deleuze, Gilles, Diferencia y repetición, p. 36.

21 Ibid. 


\section{$\S$ 3. Nueva ciencia: ¿una ciencia deleuzeana?}

El primer autor que aparece en nuestro imaginario cuando intentamos pensar una ciencia libre o liberadora de la tradición es Paul Feyerabend. Más allá de su anarquismo metodológico, este autor deja siempre la posibilidad, democratizante y productiva, de "proliferar" 22 ; sin embargo, la crítica que esgrime Feyerabend no escaparía, para Deleuze, de un tipo de ciencia extensiva: de un sistema totalizante como marco general sobre el que se presenta una oposición.

Deleuze da un paso más allá en una propuesta de ciencia y esta no se concentra ni en el método ni en el resultado, sino en la forma en que los fenómenos a explicar se conciben. En sus propios términos, está concentrado más en el campo de inmanencia detrás de las respuestas científicas, en las intuiciones que constituyen esa capa silenciosa desde donde nuestra respuesta toma forma. Su idea, entonces, es fijar una ciencia intensiva, basada en el tiempo cualitativo y la diferencia. Esto en contra de una ciencia extensiva, de tiempo cuantitativo y basada en la dialéctica negativa, exterior y artificial, es decir, a la ciencia como se ha venido trabajando desde que puso sus cimientos en la Modernidad.

Un buen ejemplo de estas nuevas formas de pensar y con ello dar cuenta de que nos inscribimos en distintos planos de inmanencia son las ciencias de sistemas complejos. La idea general es que en sistemas complejos, como la sociedad, la economía, la biología o la meteorología, las intensidades son más importantes en la explicación o en todo caso, los métodos cuantitativos no procuran mayores formas de sentir y pensar los fenómenos científicos ${ }^{23}$. Las formas no se explican por el lugar que ocupan sino por las fuerzas que la producen que son flujos constantes de materia y energía. De acá se sigue la inutilidad en ciertas ciencias sociales, como la ciencia política de tendencia empirista, en cuantificar el "avance democrático en las sociedades" mediante estadísticas de bienestar de vida o número de personas que tienen acceso al

22 Cf. Feyerabend, Paul, Contra el método, Barcelona: Ediciones Folio, 2002, p. 12.

23 Cf. Valverde, Sergio, "Historia intensiva y el nuevo materialismo: Deleuze y De Landa", en: Cuadernos digitales: publicación electrónica de historia, archivística y estudios sociales (http:// historia.fcs.ucr.ac.cr/cuadernos/c-26his.htm). Consulta: 16 de noviembre de 2015. 
voto ${ }^{24}$. Por tanto, el desplazamiento epistemológico radica en el movimiento hacia la intensidad y la naturaleza no-aritmética del tiempo: debemos atender al tiempo como intuición vital y no como intuición matemática.

Otro caso que podemos utilizar es el campo de la termodinámica. La metáfora de "la flecha del tiempo" 25 alude a que si la naturaleza se compone de dos tipos de fenómenos, los reversibles (ciclos) y los irreversibles (eventos), la termodinámica estudia estos últimos centrándose no en su repetición cuantitativa sino en su diferencia intensiva, en su devenir. Toda la ciencia clásica toma la reversibilidad como su presupuesto fundamental ${ }^{26}$ olvidándose del tiempo (cualitativo) como un elemento importante en el conocimiento. De la misma manera, las ciencias sociales y la filosofía han estado sujetas a este modo idéntico e intemporal de conocimiento, por la influencia de Platón ${ }^{27}$. Dentro de la ciencia, las leyes newtonianas, las ecuaciones maxwellianas, la relatividad de Einstein y aun la física cuántica permanecen inalteradas si introducimos una noción material, no-intuitiva del tiempo: todas llevan el sesgo de la idealización y no contemplan al fenómeno en su constante novedad. Da alcances, sin duda alguna, pero la exploración de líneas alternas también es una promesa viable.

Hay un conjunto de oposiciones que permiten clarificar cuales son las características de esta ciencia de la différence ${ }^{28}$ :

i) Inestabilidad vs. estabilidad: en la ciencia intensiva, la inestabilidad es el campo de las fuerzas. Es donde estas juegan un papel activo como diferencia de intensidades o voluntades en la configuración de la representación. La ciencia clásica depende de la estabilidad dada por los presupuestos absolutos, mientras que esta nueva ciencia puede considerarse como un campo agónistico, donde el devenir está integrado en la explicación.

24 Cf. Landa, Manuel, "Deleuze and the Open-Ended Becoming of the World", Conference "Chaos/ Control: Complexity", ZiF Bielefeld 27 de Junio, 1998 (http://www.cddc.vt.edu/ host/delanda/pages/becoming.htm). Consulta: 20 de enero de 2016.

25 Price, H., Time's Arrow and Archimedes Point, New York: Oxford University Press, 1996, p. 22.

26 Idea central que adopta la filosofía desde sus inicios: la posibilidad de retrotraer la argumentación (cf. Badiou, Alain, Condiciones., p. I5I).

27 Ibid.

28 Sobre los alcances mencionados a continuación: cf. Valverde, Sergio, op. cit. 
ii) Colapso vs. perfección en el equilibrio: para Deleuze, el equilibrio como ideal onto-teológico de la ciencia y la moral significa la muerte de la voluntad o el fin del evento. El equilibrio no se considera como un ideal regulador, más bien es la tendencia al totalitarismo, a la sentencia del orden por sobre la vida. Y lo podemos ver muy bien en los asuntos políticos internacionales. La globalización puede ser el resultado ideológico mundial del sistema económico liberal del equilibrio, aun cuando su dinámica interna financiera se asemeje a los sistemas reflexivos e irreversibles. El resurgimiento del terrorismo ante la globalización es la manifestación del colapso de un sistema de "equilibrio". Como dice Baudrillard, "es el mundo luchando consigo mismo" debido a la imposibilidad de lo universal, "donde un sistema cuyo exceso de poder crea un reto insoluble, el terrorismo responde mediante un acto definitivo que no puede ser parte del circuito del intercambio". Es decir, antes los sistemas políticos totalizantes, esos que alejan la contingencia de la vida, la subversión, los eventos internos a los países, y las luchas colectivas, funcionan como un activador del caos, de lo irrepetible y del devenir $y$ de la libertad.

iii) Apertura vs. clausura del sistema: la no-linearidad por definición no se cierra, el evento permanece abierto en sus consecuencias, influyen en ellas. Contrario al ciclo repetitivo, cerrado per se, el evento científico o social se expande por "rizomas" que no son reductibles ni a lo uno ni a lo múltiple. Un ejemplo son las revoluciones, como eventos radicales introductores de novedad, los cuales poseen consecuencias todavía perceptibles por sus repercusiones en miedos, en traumas, en fin, en intensidades no matematizables o que escapan a este intento. Muchos fenómenos, en geología, meteorología, biología, economía y sociología, emergen espontáneamente por el juego entre la intensidad abierta de diferencias. De alguna manera, es la parte activa de la materia, la que es sensible al cambio. Nuestra clásica visión esencialista ve la materia como un receptáculo inerte de formas que vienen de fuera (esencias trascendentales desde Platón hasta Husserl). En la visión intensiva, la materia posee sus propios recursos inmanentes e intensivos que generan la forma desde adentro.

De acuerdo a esta versión "no-materialista clásica" o "neo-materialista", la realidad se debe entender de manera, compleja, heterogénea y no-esencialista. 
El marco ontológico es una ontología de la diferencia, evidentemente inmanentista. Según Deleuze, la génesis de la forma no es trascendente, es inmanente a la materia misma. Al tomar el postulado de Spinoza según el cual Dios es causa inmanente del mundo, Deleuze intenta describir como todos los procesos reales provienen de la materia misma y no según una inteligencia exterior a ella. En este (neo) materialismo radical, las nociones de realidad y posibilidad se redefinen. Si el futuro es meramente la modalidad del tiempo donde las posibilidades determinadas previamente se actualizan, entonces la innovación verdadera es imposible. Deleuze, entonces, toma de Bergson el tema de la virtualidad para distinguirlo de la mera potencialidad. Según la potencia, las esencias predefinidas se actualizan según su parecido a la idea fundante. Según lo virtual, lo actual nada añade a la potencia, excepto realidad. No hay diferencia entre una esencia concebida y una esencia realizada, es decir, no hay diferencia entre realidad y pensamiento. La diferencia, por otro lado, entre lo virtual y lo real es que el parecido, o la semejanza, entre ambos, desaparece. La naturaleza de algo define su potencialidad, pero no su virtualidad. La posibilidad difiere de la virtualidad en cuanto a su relación semejante con lo real. En los sistemas reversibles hablamos de lo real y lo posible: todo lo contenido en lo real es también su posibilidad. En los sistemas irreversibles hablamos, pues, de lo actual y lo virtual para así dar campo a una ciencia que introduzca el tiempo en su interior. Esta relación entre lo virtual y lo real es de co-presencia: si la potencia es algo futuro a lo real pero contenido en ello, lo virtual es co-presente a lo real pero diferente a éste. Por ejemplo, la potencialidad de una cosa reside en que cualquiera de sus propiedades materiales se actualicen mañana: el niño deviene hombre; la semilla, flor, etc. Pero según lo virtual, hay ciertas propiedades que efectivamente corresponden al objeto, pero no son materiales. La unidad, por ejemplo, se predica de los objetos pero no es una propiedad material. Esta unidad, considerada trascendentalmente por Kant como categoría, Deleuze la considera inmanentemente como virtualidad, esto es, perteneciente a la materia misma y no puesta desde el exterior por un entendimiento trascendental. En la virtualidad, el mismo origen produce formas diferentes no contempladas por la potencia: burbujas de jabón, cristales, embriogenésis, movimientos migratorios, transacciones económicas, etc. La imposibilidad predictiva de la historia está en que los fenómenos históricos carecen de potencia, pero están llenos de virtualidad. Si se quiere trascender el positivismo histórico así como la filosofía de la historia, creo que una teoría del evento puede ser fecunda pues lidia con estos 
procesos inmanentes explicándolos y no solo describiéndolos. A diferencia de la ontología clásica, lo virtual introduce una ontología de la novedad y de la espontaneidad, alejado del causalismo filosófico que es incapaz de explicar el dinamismo inmanente de la materia.

Lo virtual es el concepto central de la ontología de la diferencia. Lo que nosotros vemos negativamente como contradicción, la diferencia la postula positivamente como novedad. Si el idealismo moderno define al mundo-en-sí como incognoscible y el relativismo lo define como construcciones mentales, sociales o lingüísticas, el realismo de Deleuze defiende la autonomía del mundo real sin adecuarlo al intelecto. El mundo es diferente del pensamiento, no adecuable ni representable por él. En épocas pasadas, esta posición estaba definida como irracionalismo, pero lo que Deleuze postula es un realismo no-esencialista donde la diferencia es el "fundamento" de la realidad: "La diferencia no es diversidad. La diversidad está dada, pero la diferencia es aquello por lo cual lo dado está dado (...). La diferencia no es un fenómeno, sino el noúmeno más cercano al fenómeno (...). Cada fenómeno se refiere a una desigualdad por la cual está condicionado (...). Todo lo que pasa y todo lo que aparece está correlacionado con órdenes de diferencias: diferencias de nivel, temperatura, presión, tensión, potencial, diferencia de intensidad”29.

A estos alcances, me gustaría incluir también otro ámbito de acción para pensar una ciencia intensiva: el estudio del lenguaje y del significado. Desde mi punto de vista, el ser humano tiene dos dimensiones: una analógica y una digital $^{30}$. La primera es el terreno de las potencias, fuerzas e intensidades, naturales y reconocibles en la rabia más turbia o en el llanto flotante de un niño. En la alegría más poderosa o en la sensación más suave que la música puede generar. Lo analógico es el espacio donde el arte fija sus estatuas, marcas sus trazos y el deseo impronunciable habita a sus anchas. Es, sin más, el terreno del significado, no uno relacional, sino el vívido, el que llena las palabras de existencia y donde todo se pone en movimiento. Es el significado que permite que una palabra usada no represente lo mismo para dos sujetos

29 Deleuze, Gilles, Diferencia y repetición, p. 61.

30 Esta distinción implica que la poesía es el arte mayor por tener un pie en lo digital y en lo analógico. $\mathrm{O}$ al menos ese es su intento. 
y esto por todas las silenciosas relaciones que convoca: aromas, formas de comportamiento, vínculos personalísimos, etc.

La segunda es el terreno del orden de la sintaxis, las reglas, las órdenes, el cálculo fijo de la lógica. Es una red que surge posterior a la primera fuerza y la encasilla, la delimita, la hace reconocer que este sacrificio solo se da en virtud de comunicarnos con otros y solo a través de esa parte de las palabras es que es posible comunicar. Es el espacio de las reglas de comportamiento y de los límites. El tiempo nos hace expertos viajeros dentro de esta fuerza. Incluso reconociendo que trastabillar no invalida este vehículo para compartir todo lo que se pueda compartir.

En este sentido, todos los desarrollos relacionados a formalizar el lenguaje, tanto la lógica simbólica como, por ejemplo, la gramática generativa de Chomsky se concentran en exponer uno de los aspectos inscritos en el plano digital. Y es más que evidente que estas empresas caen, fallan, y se equivocan cuando intentan abordar el tema del significado, el que está inscrito en las intensidades. Son sustancias distintas, ya que el significado pertenece al ámbito de lo analógico, al de fuerzas, a un campo de inmanencia distinto, donde el devenir está siendo constantemente. Justamente por ello, en la actualidad, es que se busca abordar este tema, el del significado, por otro camino, por un camino donde los sistemas autopoiéticos, los vínculos rizomáticos se despliegan y el cuerpo juega un papel central en este desarrollo. Me refiero con esto a los sistemas autopoiéticos de Maturana ${ }^{31}$, a la gramáticas cognitiva de Langacker ${ }^{32}$ y al estudio de metáforas en el lenguaje de parte de Lakoff ${ }^{33}$. En este espacio, las preposiciones por ejemplo, son componentes gramaticales ahora, pero en el pasado fueron metáforas relacionadas con el cuerpo, con la dirección, luego a través de su uso y su inclusión paulatina en la sintaxis, en lo digital, va perdiendo su significado inicial y se convierte en otra pieza de la gramática. Esta

3I Cf. Varela, Francisco y otros, "Autopoiesis: the organization of living systems, its characterization and a model", en: Biosystems, No. 5 (1974), pp. 187-196.

32 Cf. Langacker, Ronald, Cognitive grammar: a basic introduction, New York: Oxford University Press, 2008, p. 30.

33 Cf. Lakoff, George, Where mathematics comes from: how the embodied mind brings mathematics into being, New York:Basic Books, 2000, p. 15I. Para una introducción clara y expositiva a estos nuevos caminos de la lingüística véase Cuenca, María Josep y Joseph Hilferty, Introducción a la lingüística cognitiva, Barcelona: Ariel, 1999, p. 256. 
idea se conoce hace mucho tiempo pero la falta el impulso cualitativo que acá hemos comentado no ha permitido su desarrollo. Los neogramáticos indicaban que la sintaxis era la morfología del ayer, ahora a la luz de una nueva ciencia podríamos indicar que la morfología, la más cercana a lo pragmático sobre todo, es justamente la puerta abierta de lo analógico en lo digital del lenguaje.

No es posible, hasta el momento, desarrollar una ciencia del lenguaje que concilie ambas perspectivas. La razón: ambas siempre intentan tiranizar a la otra otorgándole una centralidad e importancia que no se revela a la intuición. La razón es que el lenguaje tiene las dos caras, siempre actuando, siempre haciendo lo que las reglas de su naturaleza le permiten. Atender a esos puntos de (des) encuentro son caminos pendientes en la actualidad, pero que para nuestra exposición bien ejemplifican la distinción entre las intensidades, lo cualitativo, lo analógico y lo reversible, lo cuantitativo y lo digital.

\section{§4. A modo de cierre}

A lo largo de esta breve exposición hemos podido abordar dos de los tres malentendidos que suelen perseguir la lectura de Gilles Deleuze. Nos hemos concentrado en el malentendido a) el vinculado a la ontología y hemos puesto en relieve los aportes de Bergson y Spinoza en Deleuze, principalmente a través de una concepción del Ser positivo y de una crítica al movimiento dialéctico negativo que se heredó de Hegel. Por otro lado, hemos tratado de describir las características de una ciencia intensiva, es decir, una ciencia basada en la diferencia utilizando los casos de los sistemas complejos, explicaciones en política y sobre el estudio del lenguaje. Creemos que estos puntos deben seguir siendo desarrollados y discutidos, por ello acá nos hemos remitido a una presentación superficial de los mismos para dejar en claro las intenciones del pensamiento de Gilles Deleuze. 